\title{
Anja Lorenz
}

\section{Frei lizenzierte Bildungsmaterialien (nicht nur) von der TH Lübeck}

Der stetige Zuwachs an digitalen Möglichkeiten, aber auch die zunehmende politische und gesellschaftliche Forderung nach Nachhaltigkeit bei staatlichen Investitionen in das Bildungssystem fördern die Auseinandersetzung mit Open Educational Resources (OER). Am Institut für Lerndienstleistungen (ILD) der Technischen Hochschule Lübeck (TH Lübeck) wurden zunächst mit kleinen Schritten erste Erfahrungen in der Produktion und Nutzung frei lizenzierter Materialien gemacht. Vor allem mit der Umsetzung von offenen Online-Kursen (Massive Open Online Courses, MOOCs) wurden zunehmend Kompetenzen aufgebaut und neue Partnerschaften in Projekten in und außerhalb der akademischen Bildung erschlossen.

In diesem Beitrag werden die Aktivitäten des ILD rund um freie Lernmaterialien beschrieben. Ausgehend von den Anfängen und ersten Experimenten wird dargestellt, wie die Öffnung selbst produzierter Lernmaterialien nicht nur die Sichtbarkeit und damit die Reputation des Instituts gesteigert hat, sondern auch zu neuen Partnerschaften und Projektförderungen führte.

\section{Was sind OER?}

2002 wurde die Bezeichnung Open Educational Resources erstmals von der UNESCO für die offene Bereitstellung von Bildungsressourcen verwendet. ${ }^{1}$ Im derzeit geltenden Koalitionsvertrag der auf Bundesebene regierenden Parteien CDU, CSU und SPD wurde

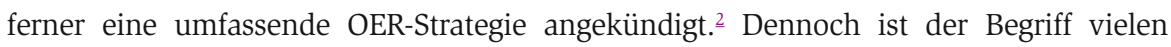
Menschen unbekannt - einschließlich Lehrkräften an Schulen oder Dozierenden an Hochschulen. Analog zu der Diskussion um Open Access für wissenschaftliche Publikationen wurden auch die Forderungen nach frei verfügbaren Bildungsmaterialien vor allem durch die Möglichkeiten einer vernetzten und digitalen Welt intensiviert.

War der Einsatz urheberrechtlich geschützten Materials in Unterricht und Lehre durch die geltende Urheberrechtsschranke in §60a des deutschen Urheberrechtsgesetzes (UrhG) in einem gewissen Rahmen bedenkenlos möglich, gelten im offenen Internet weitere Einschränkungen, die Lehrenden vielfach nicht bekannt sind. Fälle wie das sogenannte ,Cordoba-Urteil', in dem die Verwendung eines urheberrechtlich geschützten Fotos auf einer Schul-Webseite abgemahnt wurde, ${ }^{3}$ tragen einerseits zu einer Verunsicherung von Lehrenden beim Einsatz digitaler Medien bei, fördern andererseits aber auch 
die Auseinandersetzung mit urheberrechtlichen Einschränkungen und den Möglichkeiten, die OER als frei lizenzierte Materialien für den rechtssicheren Einsatz mit sich bringen.

OER sind Materialien, die zum Lernen eingesetzt werden können und die unter einer freien Lizenz stehen, damit sie verwahrt und vervielfältigt, verwendet, verarbeitet, vermischt und verbreitet werden können. ${ }^{4}$ Als freie Lizenzen werden vor allem die von der gemeinnützigen Organisation Creative Commons ${ }^{\underline{5}}$ entwickelten eingesetzt. $\underline{\underline{6}}$

\section{OER aus Lübeck: Aktivitäten des Instituts für Lerndienstleistungen}

An der TH Lübeck wurden mit dem Institut für Lerndienstleistungen und der THLübeck-Tochter oncampus GmbH bereits seit 1997 Kompetenzen und Kapazitäten für die Online-Lehre aufgebaut. Für die Virtuelle Fachhochschule (VFH), 르 einem Hochschulverbund zur gemeinsamen Bereitstellung von Online-Studiengängen, werden hier Infrastrukturen entwickelt und zentral für alle teilnehmenden Hochschulen betrieben. Mit dem Aufbau digital gestützter Lernangebote wurde das Potential zur Öffnung über die traditionelle Zielgruppe der immatrikulierten Studierenden hinaus erkannt und sukzessive auch außerhalb der hochschulinternen Systeme erprobt.

\subsection{Die Anfänge: What could possibly go wrong?}

Einer der ersten Schritte hin zu einer OER-Strategie war für die TH Lübeck gar nicht so groß: Eine Reihe der ohnehin auf YouTube gehosteten und für alle zugänglichen Lehr-

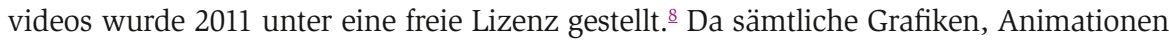
und schließlich auch die final entstandenen Videos inhouse produziert wurden, lag die Entscheidung über die Lizenz ganz beim ILD, das hierfür mit dem Wikimedia WissensWert-Preis inklusive Publikumspreis ausgezeichnet wurde. ${ }^{?}$

Eine weitere Option für die Öffnung von ohnehin produzierten Lernmaterialien bot die Mediawiki-basierte Learning Object Online Platform (LOOP) für interaktive Skripte..$^{10}$ Neben der Möglichkeit für Autor*innen von Online-Studienmodulen, ihre Inhalte unter eine freie Lizenz zu stellen, $\underline{11}$ konnten auch externe OER-Projekte wie der SchulbuchO-Mat unterstützt werden. $\underline{12}$

Zum Austausch über Best Practices - sowohl über E-Learning-Ansätze an sich, als auch über Produktion und Nutzung frei lizenzierter Lernmaterialien - fanden sich in dieser Zeit erste Communities, und so entstand auch das frei lizenzierte Lehrbuch für Lernen und Lehren mit Technologien (kurz L3T) unter Mitwirkung aus Lübeck. $\cdot \underline{13}$ 


\subsection{MOOCs made in Lübeck}

Einen erneuten Schub bekam die Öffnung digitaler Angebote der TH Lübeck durch die Produktion und den Aufbau einer Infrastruktur für MOOCs. Hierbei handelt es sich um Online-Kurse, die für alle Menschen offen bereitstehen und deswegen häufig hohe Teilnehmerzahlen aufweisen.

Durch eine Förderung durch die Possehl-Stiftung konnten 2014 der HanseMOOC produziert und nachfolgend erste Erfahrungen mit derartigen, skalierbaren Kursformaten gesammelt werden..$^{14}$ Die hierfür angepasste Moodle-Instanz führte im März 2015 zur Veröffentlichung der Lübecker MOOC-Plattform mooin,,$\frac{15}{}$ auf der die TH Lübeck eigene MOOCs hosten konnte, ohne in die Abhängigkeit von Drittplattformen zu geraten. Mit der Zeit wurde die Plattform auch für externe Partnerschaften und MOOC-Maker geöffnet: Bereits zwei Monate nach dem Launch wurde der MOOChub gegründet, $\frac{16}{6}$ über den mehrere Plattformen ihre Kurse gegenseitig verlinken und somit einer größeren Zielgruppe zugänglich machen können. Seit 2016 steht zudem externen Personen offen, ihre eigenen MOOCs auf der Plattform zu erstellen. ${ }^{17}$ Mit dem Relaunch von oncampus.de verschmolz die MOOC-Plattform endgültig mit dem (eher akademischen) Lernangebot der oncampus $\mathrm{GmbH}$.

Die Themen der MOOCs sind bedingt durch die unterschiedlichen Zielstellungen und -gruppen in den drittmittelgeförderten Projekten der TH Lübeck, aber auch durch Öffnung gegenüber anderen MOOC-Anbietenden, sehr heterogen..$^{18}$ Neben akademischen Angeboten aus dem Bereich der wissenschaftlichen Weiterbildung zu Themen wie Netzwerksicherheit, Kosten- und Leistungsrechnung oder Suchmaschinenmarketing tragen TH-Lübeck-Produktionen wie der ichMOOC, der zusammen mit den Volkshochschulen Bremen und Hamburg über das Agieren in sozialen Netzwerken gestartet wurde, oder der efiMOOC, in dem sich ehrenamtlich arbeitende Senioren für das bürgerschaftliche Engagement weiterbilden und vernetzen können, zu einem vielfältigen Programm bei. Mit Angeboten wie dem MOOC zum ,Aussprachetraining für syrische Deutschlernende‘, der gemeinsam mit Jürgen Handke von der Philipps-Universität Marburg produziert wurde, konnte die Mehrsprachigkeit und Lokalisierung der Plattform vorangetrieben werden: Texte und Medienelemente mussten auf Deutsch und Arabisch in den Kurs eingearbeitet und Navigationselemente auch für den im Arabischen gebräuchlichen rechts-nach-linksLesefluss angepasst werden. ${ }^{19}$

Mit dem Einsatz der frei lizenzierten Kurse in verschiedenen Szenarien und für verschiedene Zielgruppen wuchsen auch die Lösungen und Funktionalitäten zur Integration kompetenzgerechter Aufgaben und interaktiver Inhalte stetig an. Mit dem Lernmanagementsystem Moodle konnte auf ein breites Plugin-Angebot und auf vielfältige Erfahrungen der Community rund um die Open-Source-Software zurückgegriffen werden. Weitere Neuentwicklungen ließen sich schnell integrieren, allen voran H5P, ein 
Open-Source-Tool zur Erstellung interaktiver Inhalte..$^{20}$ Auf diese Weise kann auch die Skalierung der Plattform ohne horrende Lizenzkosten bei steigenden Nutzungszahlen vorangetrieben werden. Aktuell (Stand: 16.03.2020) sind 77.222 angemeldete Nutzer*innen auf der Plattform registriert.

\subsection{Von offenen Kursen zur schrittweisen Öffnung der Hochschule}

Wenn es keine triftigen Gründe dagegen gibt, stehen alle MOOCs der TH Lübeck unter der Creativ-Commons-Lizenz Namensnennung (CC BY). 리 Diese potentielle Möglichkeit zum Austausch von Lernmaterialien brachte, wie im Folgenden dargestellt, eine Reihe von Kooperationen hervor.

\subsubsection{MOOChub}

Bereits zwei Monate nach dem Launch der MOOC-Plattform wurde mit dem österreichischen Pendant der sogenannte MOOChub gegründet. Ziel ist es, die offenen Online-Kurse der jeweils anderen Plattform zu verlinken und somit die Bekanntheit der Kurse insgesamt zu erhöhen. Auf diesem Weg konnten zum Start der Kooperation ca. acht Prozent der externen Referrer über die gegenseitige Verlinkung festgestellt werden.2

\subsubsection{COER16}

Die offene Lizenzierung der Kurse selbst führte zu einer stetigen Verbesserung und Wiederverwendung einmal produzierter Inhalte. So wurde beispielsweise der Course zu Open Educational Resources 2013, kurz COER13, im Jahr 2016 von der TH Lübeck und den Grazer Universitäten gemeinsam neu aufgelegt, um zusätzliche Inhalte erweitert und auf den beiden Plattformen mooin und iMooX gleichzeitig durchgeführt. ${ }^{23}$

\subsubsection{OER-Festivals und OER-Awards}

Um den bis dahin noch vor allem Bottom-Up-getriebenen Aktivitäten im OERBereich eine größere Sichtbarkeit zu verschaffen, engagierten sich die oncampus GmbH bzw. die TH Lübeck als Mitausrichter*innen der OER-Festivals 2016 und 2017 in Berlin. ${ }^{24}$ Neben einem umfassenden Vortrags- und Workshop-Programm inklusive BarCamp-Format wurden vor allem die Verleihungen der OER-Awards als Auszeichnungen für preiswürdige OER-Projekte von Lübeck aus koordiniert.

\subsubsection{OERexp und JOINTLY}

Die Reputation der TH Lübeck im Bereich OER führte nicht nur mittelbar zu weiteren Projekten: Im Rahmen der Förderlinie ,OERinfo ‘ konnten 2016 zwei Projekte auf der Grundlage neuer Partnerschaften eingeworben werden: Im Rahmen der Fachfortbildung ,OER-Fachexperten' wurde der bestehende MOOC zu OER nochmals aktualisiert und mit weiteren Inhalten speziell für die Zielgruppe der freiberuflichen Trainer*innen erweitert. $\frac{25}{5}$ Ergänzend hinzu kamen Webinare, Präsenzveranstaltungen und eine Projektarbeit, mit denen nach einem Prüfungsgespräch ein Zertifikat erworben werden kann. 
Die Angebote aus dem Verbundprojekt JOINTLY는 richten sich an die OERCommunity, deren Aufgabe es im Rahmen der Förderlinie ist, Multiplikator*innen zu schulen. Die dafür benötigten Materialien und Infrastrukturen wurden zusammengetragen, gegebenenfalls weiterentwickelt und beispielsweise über das OER-Contentbuffet ${ }^{27}$ bereitgestellt.

\section{Fazit}

Die Aktivitäten des Instituts für Lerndienstleistungen im Bereich der frei lizenzierten Lernmaterialien (OER) mit Schwerpunkt auf offenen Online-Kursen (MOOCs) führte nicht nur zu nachhaltig nutzbaren Projektergebnissen, sondern auch zu weiteren Kooperationen und Projektförderungen. Auch bei Ausschreibungen, die nicht explizit OER erfordern, wurden die formulierten Absichten, Projektergebnisse frei lizenziert zu veröffentlichen, stets positiv gesehen.

So, wie der freie Zugang zu öffentlich finanzierten Forschungsergebnissen in der Open-Access-Debatte gefordert wird, engagiert sich eine wachsende OER-Community für die freie Bereitstellung staatlich finanzierter Lernmaterialien. Der gesellschaftliche Auftrag, der mit der Finanzierung aus Steuermitteln einhergeht, erhält umso mehr Gewicht, wenn die entstehenden Ergebnisse rechtssicher weiterverwendet und für neue Angebote angepasst werden können.

Aktuell lässt die im Koalitionsvertrag angekündigte OER-Strategie noch auf sich warten. Dennoch wird OER zunehmend bei Förderausschreibungen erwartet und eine Infrastruktur aufgebaut. Einige Bundesländer, wie Baden-Württemberg ${ }^{28}$ oder Bayern ${ }^{29}$ stellen bereits OER-Infrastrukturen bereit oder bauen diese wie Niedersachsen ${ }^{30}$ und Nordrhein-Westfalen ${ }^{31}$ aktuell auf. Weitere Landesportale - ob nun dediziert für OER oder gemeinsam mit anderen Bildungsangeboten - sind als Grundlage vieler Digitalisierungsprojekte im Bereich des lebenslangen Lernens zu erwarten.

Anja Lorenz ist studierte Medieninformatikerin. Sie arbeitet am Institut für Lerndienstleistungen der Technischen Hochschule Lübeck. Ihre Schwerpunkte liegen in den Bereichen E-Learning, Social Media und Open Education. Kontakt:anja.lorenz@th-luebeck.de 


\section{Anmerkungen}

Der Beitrag ist unter der Creative-Commons-Lizenz Namensnennung 4.0 international veröffentlicht. Den Vertragstext finden Sie unter: https://creativecommons.org/licenses/by/4.0/deed.de. Bitte beachten Sie, dass einzelne, entsprechend gekennzeichnete Teile des Werks von der genannten Lizenz ausgenommen sein bzw. anderen urheberrechtlichen Bedingungen unterliegen können.

1 UNESCO. Forum on the Impact of Open Courseware for Higher Education in Developing Countries (Cl-2002/C0NF.803/ CLD.1), 2002, abrufbar unter: https://unesdoc.unesco.org/ark:/48223/pf0000128515 (22.08.2020).

$\underline{2}$ Ein neuer Aufbruch für Europa. Eine neue Dynamik für Deutschland. Ein neuer Zusammenhalt für unser Land. Koalitionsvertrag zwischen CDU, CSU und SPD, 2018, abrufbar unter: https://cdn.netzpolitik.org/wp-upload/2018/02/koalitionsvertrag 2018-1.pdf (14.08.2020)

3 Europäischer Gerichtshof. Urteil des Gerichtshofs in der Rechtssache C-161/17, 2018, abrufbar unter: http://curia.europa.eu/ juris/document/document.jsf?docid=204738\&doclang=de (14.08.2020).

4 Vgl. z.B. Jöran Muuß-Merholz, Freie Unterrichtsmaterialien finden, rechtssicher einsetzen, selbst machen und teilen: Mit Online-Materialien und E-Book inside, Weinheim 2018, S. $42 \mathrm{f}$.

$\underline{5}$ https://creativecommons.org (14.08.2020).

$\underline{6}$ Zur Erläuterung sei an dieser Stelle auf Jöran Muuß-Merholz (wie Anm. 4), S. S. $47 \mathrm{ff}$. , verwiesen.

$\overline{7}$ https://www.vfh.de (26.08.2020)

$\underline{8}$ Andreas Wittke, Wir haben gewonnen - Wikimedia vergibt WissensWert Preise an oncampus, 2011, abrufbar unter: https://www.onlinebynature.com/2011/12/wir-haben-gewonnen-wikimedia-vergibt-wissenswert-preise-an-oncampus/ (14.08.2020).

9 Wikmedia. WissensWert 2011/07-Barrierefreie YouTube OER Videos, abrufbar unter: https://meta.wikimedia.org/wiki/WissensWert 2011/07-Barrierefreie YouTube OER Videos (14.08.2020).

10 https://loop.eduloop.de/ (01.03.2021).

11 Siehe z. B. Virtuelle Fachhochschule: Computerarchitektur und Betriebssysteme, abrufbar unter: https://vfhcab.eduloop.de/ loop/Computerarchitektur und Betriebssysteme (01.03.2021).

12 SCHULBUCH-O-MAT. Eine OER Schulbuch-Gemeinschaft, eine Initiative von Heiko Przyhodnik und Hans Hellfried Wedenig, 2013, abrufbar unter: http://www.schulbuch-o-mat.de/ (14.08.2020)

13 Martin Ebner, Sandra Schön, Lehrbuch für Lernen und Lehren mit Technologien, Bad Reichenhall 2013, abrufbar unter: https://l3t.eu/ (14.08.2020).

14 Oncampus: Die Welt der Hanse (\#HanseMO0C), 2014, abrufbar unter: https://www.oncampus.de/weiterbildung/moocs/ hanse-mooc (14.08.2020)

15 Anja Lorenz, Andreas Wittke, Thomas Muschal, Farina Steinert, From MOODLE to MOOIN: Development of a MOOC platform, in: Proceedings Papers of the European MOOCs Stakeholder Summit 2015 (EMOOCs2015), 18.-20. Mai 2015, Mons 2015, S. $102-106$.

16 https://www.oncampus.de/moochub (14.08.2020).

$\frac{17}{17}$ https://www.oncampus.de/weiterbildung/moocs/mooin-maker (14.08.2020)

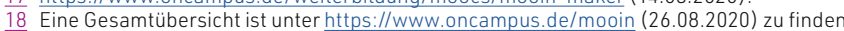

19 Anja Lorenz, Andreas Wittke, Linda Wulff, \#refugeesWelcome an der Fachhochschule Lübeck, in: Nicolas Apostolopoulos, Wolfgang Coy, Karoline von Köckritz, Ulrike Mußmann, Heike Schaumburg, Andreas Schwill (Hg.), Tagungsband GML2: Grundfragen Multimedialen Lehrens und Lernens, Die offene Hochschule: Vernetztes Lehren und Lernen, Münster 2016, S. 236-246.

$\underline{20}$ https://h5p.org/ (26.08.2020)

21 Die Fachhochschule Lübeck stellt ihre OER-Strategie auf die "Creative Commons Lizenz ,CC-BY"' um. Freie Bildung für alle mit Open Educational Resources (OER), abrufbar unter: https://www.presseportal.de/pm/116119/2952828 (14.08.2020).

22 Martin Ebner, Anja Lorenz, Elke Lackner, Michael Kopp, Swapna Kumar, Sandra Schön, Andreas Wittke, How OER Enhances MOOCs - A Perspective from German-Speaking Europe, in: Mohamed Jemni, Kinshuk, Mohamed Koutheair Khribi (Hg.), Open Education: from OERs to MOOCs. Lecture Notes in Educational Technology, 2016, S. 205-220, abrufbar unter: https://doi.org/10.1007/978-3-662-52925-6 11.

23 Martin Ebner, Anja Lorenz, Sandra Schön, Andreas Wittke, Offene Lizenzen als Treiber für neuartige Kooperationen und Innovationen in der Bildung, in: Josef Wachtler, Martin Ebner, Ortrun Gröblinger, Michael Kopp, Erwin Bratengeyer, Hans-Peter Steinbacher, Christian Freisleben-Teutscher, Christine Kapper ( $\mathrm{Hg}$.), Digitale Medien: Zusammenarbeit in der Bildung (Medien in der Wissenschaft/71), Münster/New York 2016, S. 55-64.

24 \#OERde16 - Das OER-Festival 2016, abrufbar unter: https://open-educational-resources.de/veranstaltungen/16/; OERinfo. (2017). \#OERde17 - Das OER-Festival 2017, abrufbar unter: https://open-educational-resources.de/veranstaltungen/17/ (beide 14.08.2020)

25 BDVT e.V., \& TH Lübeck. OER-Fachexperten - Open Educational Resources in der Weiterbildung, abrufbar unter: https://www.oer-fachexperten.de/ (14.08.2020).

26 https://jointly.info/ (14.08.2020).

27 OER-Contentbuffet. OER für OER: Material für OER-Einführung, -Training und -Community-Arbeit, abrufbar unter: https://oer-contentbuffet.info/edu-sharing/components/oer (14.08.2020).

28 Zentrales Repositorium für Open Educational Resources der Hochschulen in Baden-Württemberg, abrufbar unter: https://www.oerbw.de/ (14.08.2020).

29 https://oer.schule/ (14.08.2020).

$\underline{30}$ TIB Hannover. OER-Portal Niedersachsen, abrufbar unter: https://www.tib.eu/de/forschung-entwicklung/projektuebersicht/projektsteckbrief/oer-portal-niedersachsen/ (14.08.2020).

31 DigitaleHochschuleNRW, abrufbarunter: https://www.land.nrw/de/pressemitteilung/digitale-hochschule-nrw-land-stelltweichen-fuer-aufbau-des-landesportals-fuer (14.08.2020) 